\title{
Correcting the quantum clock: conditional sojourn times
}

\author{
S. Anantha Ramakrishna* and N. Kumar ${ }^{\dagger}$ \\ Raman Research Institute, C.V. Raman Avenue, Bangalore 560 080, India
}

\begin{abstract}
Can the quantum-mechanical sojourn time be clocked without the clock affecting the sojourn time? Here we re-examine the previously proposed non-unitary clock, involving absorption/amplification by an added infinitesimal imaginary potential $\left(i V_{i}\right)$, and find it not to preserve, in general, the positivity of the sojourn time, conditional on eventual reflection or transmission. The sojourn time is found to be affected by the scattering concomitant with the mismatch, however small, due to the very clock potential $\left(i V_{i}\right)$ introduced for the purpose, as also by any prompt scattering involving partial waves that have not traversed the region of interest. We propose a formal procedure whereby the sojourn time so clocked can be corrected for these spurious scattering effects. The resulting conditional sojourn times are then positive definite for an arbitrary potential, and have the proper high- and low-energy limits.
\end{abstract}

PACS Number(s): 03.65.-w, 42.25.Dd, 73.40.-c

The time scales associated with the motion of a deformable object, such as a quantum-mechanical wave packet, scattered by a potential are operationally not context-free and raise some fundamental questions of interest for mesoscopic systems (for recent reviews see [1] 3). In the present work, we will be interested in a physically relevant time scale that may aptly be called the (conditional) sojourn time which literally measures the time of sojourn of a particle in the spatial region of interest, under given conditions of scattering. Thus, one speaks of the conditional sojourn time - conditional on eventual reflection or transmission in the 1-D case. The unconditional sojourn time, irrespective of eventual reflection/transmission is then the dwell time. (We could also generalize the sojourn time to include the dwell time for a particle initially prepared in a spatially confined state - this covers the decay time of a metastable state.) Operationally, the sojourn time can be defined meaningfully by invoking a mathematical artifice called the "clock" involving, e.g. the attachment of an extra degree of freedom that co-evolves with the sojourning particle. The evolution may be a periodic one as in the case of the unitary Larmor clock [4,5] that involves the precessional angle accumulated by a spin associated with the particle in an infinitesimal magnetic field introduced for this purpose. Another 'crossover clock' involves the timeharmonic modulation of the scattering potential. Here the timescale of traversal is identified with a certain adiabatic to non-adiabatic crossover phenomenon that occurs when the traversal time matches the period of modulation [6]. Both of the above 'clocks' yield a timescale $\tau_{B L}=m L / \hbar \kappa$, called the Büttiker-Landauer time for tunneling through a nearly opaque rectangular barrier of width $L$, where $\hbar \kappa$ is the magnitude of the imaginary momentum under the potential barrier. There is yet another 'clock', the e-folding 'non-unitary' clock [7 9], wherein an infinitesimal imaginary potential is introduced over the spatial interval of interest, and the conditional sojourn time for reflection/transmission is then calculated as the derivative of the logarithm of the reflection $\left(|R|^{2}\right)$ / the transmission $\left(|T|^{2}\right)$ coefficient with respect to the imaginary potential, in the limit of the latter tending to zero, i.e., as $\tau_{s}^{R}=\hbar / 2 \lim _{V_{i} \rightarrow 0} \partial \ln |R|^{2} / \partial V_{i}$ and $\tau_{s}^{T}=\hbar / 2 \lim _{V_{i} \rightarrow 0} \partial \ln |T|^{2} / \partial V_{i}$, respectively. Recently, this was used by us successfully to calculate the probability distribution of the sojourn time for reflection from a long one-channel random potential [9], where the reflection coefficient is unity with probability one. The above clocks, namely the periodic Larmor clock and the e-folding non-Unitary clock, however, do not yield a positive-definite sojourn time in general. Thus for instance, the conditional sojourn time, for reflection say, so obtained turns out to be negative for certain deterministic potentials [7, 3]. Two explicit cases for which we have verified this negativity are (i) the local sojourn time for reflection in a sub-interval $\left[x_{0}, x_{0}+\delta\right]$ of a rectangular barrier, as in in Fig. 1(a) (even though the total sojourn time in the entire potential region, i.e., in the inteval $(-L / 2, L / 2)$, remains positive), and (ii) the total sojourn time for reflection from a $\delta$-dimer potential consisting of two unequal $\delta$-potentials separated by a distance $L$. Basically, under certain conditions, the absorptive (amplifying) potential can counter-intuitively increase (decrease) the reflection or the transmission - a manifestation of the Borrmann effect well known in the context of X-ray scattering [10]. The dwell time, however, always stays positive.

Now, admittedly, the conditional sojourn time is not an observable in the strict sense of quantum mechanics inasmuch as there is no self-adjoint operator corresponding to it with the sojourn time as its eigenvalue [11]. To the best of our knowledge, at least, no such operator has been constructed satisfactorily so far. This, however, does not diminish the importance of having a timescale that conforms to our classical intuition of the time of sojourn determined dynamically for the problem at hand. But, for this we need to have a prescription for calculating the sojourn time that yields a reasonable value so as 
to be useful as an estimate. Our criteria for the above are that the sojourn time so calculated should be (i) real and positive-definite (unlike the phase delay time of Wigner 112 which can be of either sign), (ii) additive for nonoverlapping spatial intervals, and (iii) tend to the proper classical limits (i.e., at high energies). Thus, even if not observable as an operator in quantum mechanics, it shall be a calculable quantity in terms of which one can discuss timescales (fast or slow) of physical processes of interest in mesoscopic systems. In the following, we have explicitly constructed precisely such conditional sojourn times for scattering in 1-D.

(a)

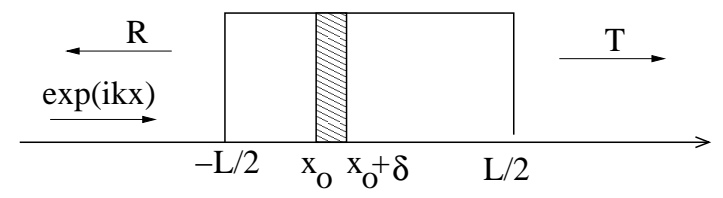

(b)
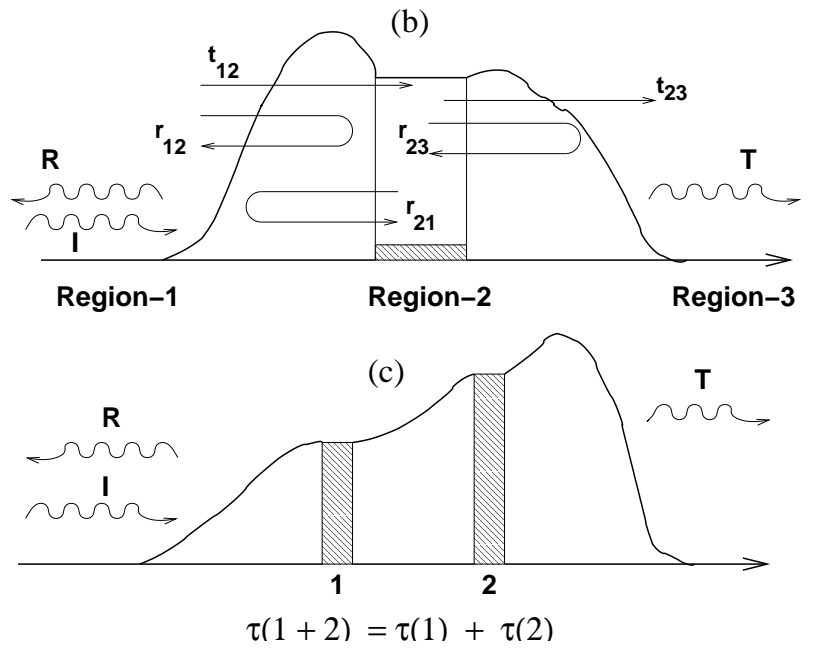

FIG. 1. The potentials considered here (a)The rectangular barrier (b)The region of interest in a region of constant potential is bounded by two arbitrary potentials whose scattering matrices are shown and (c) Shows two such regions, the local sojourn times of which add up to give the total sojourn time. The hatched region indicates the presence of the clock potential $\left(i V_{i}\right)$.

We begin by noting a rather subtle problem associated with the 'non-unitary' clock, as also with the Larmor clock, namely that the very clocking mechanism affects the sojourn time to be clocked, and it does so finitely even as the perturbing clock potential is taken to be infinitesimally small. We, therefore, re-examine the non-unitary clock so as to identify the 'spurious' perturbing terms responsible for the unphysical negativity of the conditional sojourn times. We then propose a formal and rather simple procedure for extracting the correct sojourn time. We find that the conditional sojourn time, thus corrected, is indeed positive definite and satisfies all our criteria of reasonableness. Our main results for the conditional sojourn times in a 1-D spatial interval $(\Delta)$, where the scattering potential $\left(V_{r}\right)$ is taken to be flat (constant) are

$$
\begin{aligned}
& \tau_{s}^{T}\left(E>V_{r}\right)=\hbar \Delta / 2 \lim _{\xi \rightarrow 0} \partial \ln \left|T\left(V_{i}=0, \xi\right)\right|^{2} / \partial \xi, \\
& \tau_{s}^{T}\left(E<V_{r}\right)=i \hbar \Delta / 2 \lim _{\xi \rightarrow 0} \frac{\partial}{\partial \xi} \ln \left[\frac{T\left(V_{i}=0, \xi\right)}{T^{*}\left(V_{i}=0, \xi\right)}\right], \\
& \tau_{s}^{R}\left(E>V_{r}\right)=\tau_{s}^{T}\left(E>V_{r}\right)+\tau_{S C}, \\
& \tau_{s}^{R}\left(E<V_{r}\right)=\tau_{s}^{T}\left(E<V_{r}\right)+\tau_{B L} .
\end{aligned}
$$

Here $E$ is the energy of the wave, $\xi=V_{i} \Delta$ and $\tau_{S C}$ is the semi-classical time in the high energy, i.e., $\tau_{S C}=$ $m \Delta / \hbar k$. The above times are found to be positive, and also additive, and, therefore, can be used to derive the conditional sojourn times for an arbitrary potential inasmuch as the latter can be approximated as piece-wise constant.

In the following, we will consider the case of transmission for the above-the-barrier wave energy (nontunneling) as also for the sub-barrier wave energy (tunneling) separately. The case of reflection, where a further logical refinement of our procedure is required, will be considered separately later.

The case of wave propagation, $E>V_{r}$ : Let us first consider the case of propagation (non-tunneling). For this, we calculate the total transmission and reflection amplitudes from the multiple reflections arising from the interfaces of the barrier as shown in Fig. 11(b). In the case of propagation we obtain 13

$$
\begin{aligned}
T & =t_{12} t_{23} e^{i k^{\prime} L}+t_{12} r_{23} r_{21} t_{23} e^{3 i k^{\prime} L} \\
& +t_{12} r_{23} r_{21} r_{23} r_{21} t_{23} e^{5 i k^{\prime} L}+\cdots \\
R & =r_{12}+t_{12} r_{23} t_{21} e^{2 i k^{\prime} L}+t_{12} r_{23} r_{21} r_{23} t_{21} e^{4 i k^{\prime} L}+\cdots
\end{aligned}
$$

where $k^{\prime}=\sqrt{2 m\left(E-V_{r}-i V_{i}\right)} / \hbar$ and, $r_{12}, r_{23}, r_{21}$ and $t_{12}, t_{23}, t_{21}$ are the reflection and the transmission amplitudes at the respective interfaces (See Fig. 1(b)). The transmission coefficient has a generic form $T=\sum_{k} A_{k} e^{i \phi_{k}} e^{\alpha_{k} L}$, where $A_{k}, \phi_{k}$ and $\alpha_{k}$ are real numbers representing the amplitude, phase and the growth of the partial waves. Consider now the conditional sojourn time given by the non-unitary clock as

$$
\begin{aligned}
\tau_{s}^{T} & =\lim _{V_{i} \rightarrow 0} \frac{\hbar}{2 E} \frac{1}{|T|^{2}} \frac{\partial}{\partial V_{i}}\left[\sum_{k} A_{k}^{2} e^{2 \alpha_{k} L}\right. \\
& \left.+\sum_{k \neq l} A_{k} A_{l} e^{i\left(\phi_{k}-\phi_{l}\right)} e^{\left(\alpha_{k}+\alpha_{l}\right) L}\right] .
\end{aligned}
$$

The imaginary part $i V_{i}$ of the clock potential modifies the reflection/transmission coefficients $\left(r_{j k}, t_{j k}\right)$ at the interfaces, where there is mismatch due to the imaginary clock potential. Now, the derivative with respect to the imaginary potential would cause terms of first order in $V_{i}$ to contribute to $\tau_{s}^{T, R}$, even in the limit of an infinitesimal potential $V_{i} \rightarrow 0$. Thus, the clock modifies 'spuriously' 
the propagation of the wave itself in a non-trivial manner, in addition to causing the amplification or attenuation of the wave for which it was introduced.

This analysis immediately suggests the key to correcting the quantum clock for the 'spurious' scattering. The whole point is that the presence of the imaginary potential modifies the reflection and the transmission coefficients at any point where the imaginary potential changes abruptly, even if infinitesimally. We have to, therefore, devise a method by which the clock potential $\left(i V_{i}\right)$ causes only the intended effect (amplification/absorption) without causing the 'spurious' scattering, i.e., it must be well apodized. A little thought of the perturbative structure of the scattering processes should convince one that the clock related growth/attenuation would only involve the paired combination $V_{i} \Delta$ ( $\Delta$ being the spatial interval of interest) while the 'spurious' scattering would involve unpaired $V_{i}$. This motivates the following formal procedure to eliminate the 'spurious' effects. Treating $V_{i}$ and $V_{i} \Delta \equiv \xi$ formally as independent variables, we keep $\xi$ constant and let $V_{i} \rightarrow 0$ in the expression for $T$. The transmission sojourn time is then obtained as

$$
\tau_{s}^{T}=\hbar \Delta / 2 \lim _{\xi \rightarrow 0} \partial \ln \left|T\left(V_{i}=0, \xi\right)\right|^{2} / \partial \xi .
$$

The same result is obtained also by considering the transfer matrices that explicitly suppress the 'spurious' scattering due to the clock potential $i V_{i}$.

Using either of the procedures, the local transmission sojourn time for the rectangular barrier region in Fig. 1. (b) can now be calculated. Thus, for the case of propagation $\left(v_{r}<1\right)$, we have

$$
\frac{\tau_{s}^{T}}{\tau_{B L}}=\frac{\left(1-\left|r_{21} r_{23}\right|^{2}\right)}{1+\left|r_{21} r_{23}\right|^{2}-2 \Re\left(r_{21} r_{23} e^{2 i k_{r} L}\right)}
$$

where $\Re$ is the real part, $k_{r}=\sqrt{2 m\left(E-V_{r}\right)} / \hbar$, and the $r_{j k}$ and $t_{j k}$ are the scattering amplitudes as before but with $V_{i}=0$. We note that since $\left|r_{j k}\right|<1$ for any real potential, the above sojourn time for transmission is always positive. For the case of the symmetric rectangular barrier $\left[r_{21}=r_{23}=\left(k-k_{r}\right) /\left(k+k_{r}\right)\right]$, we explicitly obtain

$$
\frac{\tau_{s}^{T}}{\tau_{B L}}=\frac{2\left(2-v_{r}\right) p}{4-4 v_{r}+v_{r}^{2} \sin ^{2}(p k L)}
$$

where $v_{r}=V_{r} / E$ and $p=\sqrt{1-v_{r}}$. In Fig. 2, we have shown plots of the transmission sojourn time for a rectangular symmetric barrier as a function of the strength of the scattering potential (for $v_{r}<1$ ).

We note that the expression given by equation (9) holds for a general class of 1-D problems. This is because the $r_{j k}$ can be the scattering matrix for any arbitrary potential, with the only condition that the real potential within the sub-interval, where we seek the time of sojourn, should be constant (see Fig. 11(b)). This is, however, not a real restriction as it can be straight-forwardly verified that the local sojourn times for traversal in different parts of the potential add up to give the total sojourn time (a schematic is shown in Fig. 11(c)). Since any arbitrary potential can be constructed out of piecewise constant potentials (in the limit of the width going to zero), we realize that the sojourn time for transmission given by this procedure is positive definite for any arbitrary potential.

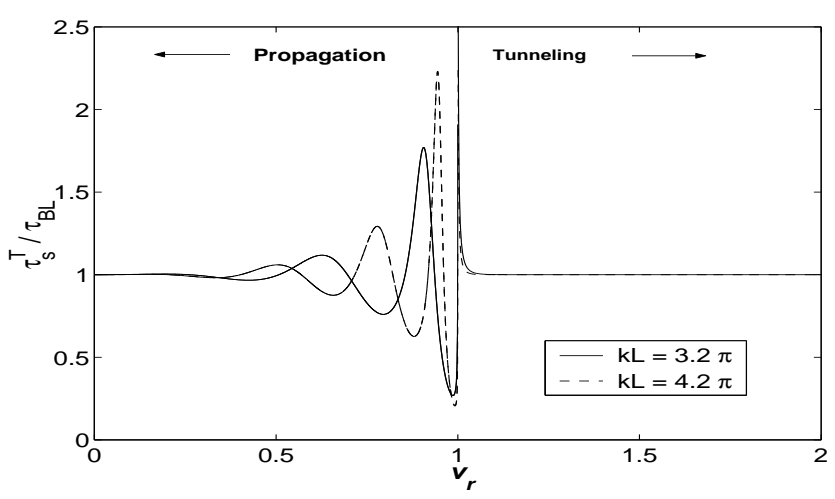

FIG. 2. The corrected sojourn times for transmission versus (a) $v_{r}=V_{r} / E$ for the rectangular barrier The times are normalized with respect to the Büttiker-Landauer traversal times $\left(\tau_{B L}\right)$.

The case of wave tunneling, $E<V_{r}$ : For the case of tunneling, we note that the wavevector becomes imaginary under the barrier. The real part of the potential sets its own length scale for the exponential decay / growth with distance under the barrier. Essentially, the roles of the real part and the imaginary part of the potential get interchanged. The imaginary part, to first order in $V_{i}$, causes an oscillation of the wave function with distance. Thus, the paired combination $\xi=V_{i} \Delta$, will now affect the phase of the wave, rather than its amplitude. Proceeding as before, we now, get the corrected sojourn time for transmission in the case of tunneling as the derivative of the phase with respect to the paired combination $\xi=V_{i} \Delta$ :

$$
\tau_{s}^{T}\left(v_{r}>1\right)=i \hbar \Delta / 2 \lim _{\xi \rightarrow 0} \frac{\partial}{\partial \xi} \ln \left[\frac{T\left(V_{i}=0, \xi\right)}{T^{*}\left(V_{i}=0, \xi\right)}\right] .
$$

For the general case, as shown in Fig. 11(b), we obtain the sojourn time of traversal as

$$
\frac{\tau_{s}^{T}}{\tau_{B L}}=\frac{\left(1-\left|r_{21} r_{23}\right|^{2} e^{-4 k_{r} L}\right)}{1+\left|r_{21} r_{23}\right|^{2} e^{-4 k_{r} L}-2 \Re\left(r_{21} r_{23}\right) e^{-2 k_{r} L}},
$$

where $k_{r}=\sqrt{2 m\left(V_{r}-E\right)} / \hbar$ now. We note that this traversal time is positive definite for any arbitrary potential. Again, as before, the local sojourn times in different parts of the potential add up to give the total sojourn time. For the case of the rectangular barrier we obtain 


$$
\frac{\tau_{s}^{T}}{\tau_{B L}}=\frac{\left(1-e^{-4 k_{r} L}\right)}{1+e^{-4 k_{r} L}-2\left[1-8\left(v_{r}-1\right) / v_{r}^{2}\right] e^{-2 k_{r} L}} .
$$

The sojourn time is plotted in Fig. 2 (for $\left.v_{r}>1\right)$. For an opaque barrier $\left(L \gg k_{r}^{-1}\right.$ or $\left.v_{r} \gg 1\right)$, i.e, in the low energy limit, the sojourn time in the above expressions tends to the Büttiker-Landauer traversal time for tunneling $\left(\tau_{s}^{T} \rightarrow \tau_{B L}\right)$. Finally, regarding the local sojourn time in any part of the rectangular barrier, we find that the ratio of the time spent in the interval $\left[x_{0}, x_{0}+\Delta\right]$ to the time spent in the entire barrier is $\Delta / L$, irrespective of the location $x_{0}$, as is also the case for the $\delta$-dimer potential. We conclude that in these cases the wave spends equal amounts of time in equal intervals of the barrier region.

The conditional sojourn time for reflection: We now consider the sojourn time for reflection for the case of over-the-barrier propagation as well as for the sub-barrier tunneling. If we look at the partial wave expansions for the transmission and the reflection amplitudes in Eqn.(6), we would realize one essential difference between the transmission and the reflection. All the partial waves of the transmitted wave sample the region of interest and correspondingly pick up the paired combination $\xi=V_{i} \Delta$ in the amplitude (i.e., the magnitude or the phase). In the case of reflection, however, there is a partial wave amplitude corresponding to the reflection from the front edge of the potential upto the region of interest (see Fig. 11(b)), due to the element $r_{12}$ in the multiple wave expansion that never samples the region of interest where the imaginary clock potential is introduced. This part corresponds to the prompt part of the reflection. Now, it is clear from the above expressions that this partial wave interferes with the rest of the partial waves, and thus affects the sojourn time to be clocked. Arguably, if this prompt partial wave never enters the region where the imaginary potential is applied, then the weightage corresponding to this partial wave should be eliminated out of reckoning. This seems reasonable to us at least in the sense of naive realism. This can be accomplished by explicitly removing the term $r_{12}$ in the right hand side of Eqn.(6) in the 1D case. Thus, we obtain the sojourn time for reflection (for $E>V_{r}$ as well as for $E<V_{r}$ ) as

$$
\begin{aligned}
& \tau_{s}^{R}\left(E>V_{r}\right)=\tau_{s}^{T}\left(E>V_{r}\right)+\tau_{S C}, \\
& \tau_{s}^{R}\left(E<V_{r}\right)=\tau_{s}^{T}\left(E<V_{r}\right)+\tau_{B L} .
\end{aligned}
$$

The reflection time in this interpretation is the sum of the transmission time and a propagation time across the sub-interval. Consequently it is always greater than the transmission sojourn time. But now the reflection time is also positive definite.

In conclusion, we have pointed out that the nonunitary clock involving the imaginary potential $\left(i V_{i}\right)$ can lead to a negative conditional sojourn time for nonrandom potentials. This negativity can be traced to the spurious scattering caused by the very clock potential introduced for clocking the sojourn time through coherent amplification/attenuation. A simple, formal mathematical procedure has been given for eliminating the effects of this spurious scattering. In the case of reflection, we further need to remove the prompt part of the reflection. With these corrections, the conditional sojourn times are found to be positive definite and additive, in general. We also find that the thus corrected non-unitary clock yields the transmission sojourn time with the proper low-energy limit in agreement with the Büttiker-Landauer traversal time. This problem of the clocking mechanism affecting the time to be clocked is not special to the non-unitary clock alone. It also affects the Larmor clock and possibly every clock where the perturbation due to the clock mechanism couples to the Hamiltonian. Finally, the conditional sojourn time proposed here, while not an observable in the sense of quantum mechanics, is a calculable intermediate quantity (like the matrix element for a transition), and is practically useful in deciding for or against certain conditions of rapidity. Moreover, it is reasonable in that it is real, positive, calculable, causally related to the region of interest, and has the correct classical limit.

[1] Th. Martin and R. Landauer, Rev. Mod. Phys. 66, 217 (1994).

[2] R.Y. Chiao and W. Steinberg, in Progress in Optics, Vol. 37, edited by E. Wolf (Elsevier, Amsterdam, 1997).

[3] E.H. Hauge and J.A. St $\phi$ vneng, Rev. Mod. Phys. 61, 917 (1989).

[4] A. I. Baź, Sov. J. Nucl. Phys. 4, 182 (1967).

[5] M. Büttiker, Phys. Rev. B27, 6178 (1983).

[6] M. Büttiker and R. Landauer, Phys. Rev. Lett. 49, 1739 (1982).

[7] Golub et al., Phys. Lett. A 148, 27 (1990).

[8] M. Büttiker, Electronic properties of multilayers and lowdimensional semiconductor structures, edited by J.M. Chamberlain et al. (Plenum Press, New York, 1990).

[9] S. Anantha Ramakrishna and N.Kumar, Phys. Rev. B61, 3163 (2000); C.W.J. Beenakker, K.J.H. van Bemmel and P. Brouwer, Phys. Rev. E60, R6313 (1999).

[10] G. Borrman, Z. Phys. 42, 157 (1941); For an optical analogue, see K.A. suresh in Liquid crystals in the nineties and beyond, ed. Satyen Kumar (world Scientific, singapore, 1995).

[11] G.R. Allcock, Ann. Phys. 53, 253 (1969); 286 (1969); 311 (1969).

[12] E. P. Wigner, Phys. Rev. 98, 145 (1955).

[13] M. Born and E. Wolf, Principles of Optics, 6th. Ed., (Pergamon Press, London, 1989). 